\title{
Laboratory-based grain-shape models for simulating dust infrared spectra
}

\author{
H. Mutschke ${ }^{1}$, M. Min ${ }^{2}$, and A. Tamanai ${ }^{1}$ \\ 1 Astrophysikalisches Institut und Universitäts-Sternwarte (AIU), Schillergäßchen 2-3, 07745 Jena, Germany \\ e-mail: mutschke@astro.uni-jena.de \\ 2 Astronomical Institute Anton Pannekoek, University of Amsterdam, Kruislaan 403, 1098 SJ Amsterdam, The Netherlands
}

Received 3 April 2009 / Accepted 16 June 2009

\section{ABSTRACT}

\begin{abstract}
Context. Analysis of thermal dust emission spectra for dust mineralogy and physical grain properties depends on comparison spectra, which are either laboratory-measured infrared extinction spectra or calculated extinction cross sections based on certain grain models. Often, the agreement between these two kinds of spectra, if available, is not yet satisfactory because of the strong influence of the grain morphology on the spectra.

Aims. We investigate the ability of the statistical light-scattering model with a distribution of form factors (DFF) to reproduce measured infrared dust extinction spectra for particles that are small compared to the wavelength, i.e. in the size range of $1 \mu \mathrm{m}$ and smaller.

Methods. We take advantage of new experimental spectra measured for free particles dispersed in air with accompanying information on the grain morphology. For the calculations, we used DFFs that were derived for aggregates of spherical grains, as well as for compact grain shapes corresponding to Gaussian random spheres. In addition we used a fitting algorithm to obtain the best-fit DFFs for the various laboratory samples. In this way we can independently derive information on the shape of the grains from their infrared spectra.

Results. With the DFF model, we achieve an adequate fit of the experimental IR spectra. The differences in the IR band profiles between the spectra of particulates with different grain shapes are simply reflected by different DFFs. Irregular particle shapes require a DFF similar to that of a Gaussian Random Sphere with $\sigma=0.3$, whereas roundish grain shapes are best fitted with that of a fractal aggregate of $D_{\mathrm{f}}=2.4-1.8$. The fitted DFFs generally reproduce the measured spectral shapes quite well. For anisotropic materials, different DFFs are needed for the different crystallographic axes. The implications of this finding are discussed.

Conclusions. The use of this model could be a step forward toward more realistic comparison data in infrared spectral analysis of thermal dust emission spectra, provided that these spectra are dominated by emission from submicron grains.
\end{abstract}

Key words. infrared: general - methods: data analysis - methods: laboratory - circumstellar matter

- planetary systems: protoplanetary disks

\section{Introduction}

The analysis of mid-infrared dust emission spectra from stellar outflows, circumstellar disks, and other objects provides information about the dust mineralogy, grain sizes, and temperatures, hence, about physical and chemical conditions in the respective environments. The information about the dust grain properties is based on the lattice vibrational bands of the dust particles that mainly occur in the $10-50 \mu \mathrm{m}$ wavelength range and that dominate the thermal emission of warm dust. Detailed analyses of, e.g., Spitzer IRS, and ground-based mid-infrared spectra including interferometric data (VLTI/MIDI) have been published in the last years for dust emission from accretion disks (e.g. van Boekel et al. 2004), debris disks (e.g. Lisse et al. 2007), and comets (e.g. Lisse et al. 2006).

The analyses are often performed by applying $\chi^{2}$ fits of a linear combination of either calculated or measured spectra of dust grains to the observed spectra. Both approaches have recently been improved by developing (1) new theoretical models (Min et al. 2005, 2006) for calculating dust spectra and (2) a new experimental method for measuring spectra of particles dispersed in air (Tamanai et al. 2006a,b), which avoids the influence of an embedding material on experimental infrared extinction spectra.
These spectroscopic measurements of dust particles in aerosol also allow the investigation of the actual morphology of the aerosol particles by filtering and subsequent scanning electron microscope (SEM) imaging. Thus, the influence of morphological particle characteristics on the dust spectra can be studied in detail. Tamanai et al. (2006b) and Tamanai et al. (2009b) have reported a strong dependence of the measured profiles of the infrared bands on grain shape and agglomeration. Although the influence of the grain morphology on dust vibrational bands has been known for many years (Bohren \& Huffman 1983), these effects have not been systematically studied in experiments before.

Furthermore, Tamanai et al. (2006b) demonstrated that the band profiles predicted by the currently used models often differ considerably from the experimentally measured ones. The reason is that most of these models such as Mie theory, CDE (Bohren \& Huffman 1983), and DHS (Min et al. 2005) are not flexible enough to take morphological effects sufficiently into account. Since this is important for interpretating astronomical spectra, we aim at (a) a clarification of typical properties of measured infrared band profiles depending primarily on the grain shape, and (b) the investigation of the ability of an alternative theoretical model to take the shape effects into account. 
For this purpose, we compare a selection of aerosolmeasured spectra of oxides and silicates for two different classes of grain morphologies with calculated spectra using the theoretical approach of a form factor distribution (Min et al. 2006). Both the experiments used here and the theory are restricted to particles small compared to the wavelength. For infrared wavelengths, this means a restriction to particles in the size range of approximately $1 \mu \mathrm{m}$ and smaller. Consequently, the applicability of our results will be limited to interpreting emission (or absorption) spectra from cosmic environments where such grains are present. If larger grains provide a strong contribution to the emission cross section, the spectrum will be influenced by grain size effects that cannot be treated by the present approach. This is especially the case when growth or radiation effects have removed submicron grains, such as in evolved protoplanetary disks and debris disks around luminous stars.

The theoretical model and the particulate materials are introduced in the next two sections, before we compare the experimental and the simulated spectra in Sect. 4. Section 5 summarizes the results.

\section{DFF model for spectra simulation}

The distribution of form factors model (DFF model, Min et al. 2006) is a statistical approach valid for ensembles of particles that are small compared to the wavelength (Rayleigh limit). It describes the averaged extinction (absorption, emission) cross section $\left\langle C_{\text {ext }}\right\rangle$ per unit volume of such a particulate by the integral over a continuous distribution of dipole polarizabilities depending on the dielectric function of the particulate material relative to its environment $\varepsilon=\varepsilon_{\mathrm{p}} / \varepsilon_{\mathrm{e}}$ and a depolarization factor or form factor $L$, which is defined in the range $0 \leq L \leq 1$ (Eq. (1)). $P(L)$ is the distribution of form factors over this range, which contains the information on the geometrical properties of the particles.

$$
\frac{\left\langle C_{\text {ext }}\right\rangle}{V}=\frac{2 \pi}{\lambda} \int_{0}^{1} \frac{P(L)}{1 /(\varepsilon-1)+L} \mathrm{~d} L
$$

Min et al. (2006) have demonstrated that a distribution $P(L)$ (hereafter called the DFF) can be calculated for each particle shape, which gives a correct representation of the extinction cross section of that particle, provided that a spatial discretization with sufficient accuracy is possible. For a distribution of shapes, the individual DFFs have to be averaged.

Another nice property of the DFF approach, which allows very intuitive understanding of the simulated spectra, is that major contributions to the integral occur when

$\varepsilon \sim 1-1 / L$

(so-called surface modes, see Bohren \& Huffman 1983). This is indeed the case in strong lattice vibration bands, where the real part of $\varepsilon$ takes a range of negative values (depending on wavelength), while the imaginary part is comparably small. Thus, relation (2) links the DFF directly to the extinction at corresponding spectral positions; e.g., for spherical grains $(P(L)=\delta(L=$ $1 / 3)$ ), the extinction cross section spectrum will show a single resonance at the wavelength where $\varepsilon=-2$. Assuming a simple Lorentzian behavior of the dielectric function, the DFF at low $L$ values is related to the strength of absorption at wavelengths longward of the sphere resonance $(\operatorname{Re}(\varepsilon)<-2)$, whereas the DFF at larger $L$ is related to absorption at shorter wavelengths (lower negative $\operatorname{Re}(\varepsilon)$ values). We illustrate this in Fig. 2, and for a more precise treatment in the complex $\varepsilon$ plane see Min et al. (2006).

The often used continuous distribution of ellipsoids model (CDE, Bohren \& Huffman 1983) can be considered a special case of the DFF model with $P(L)=2(1-L)$. Min et al. (2006) have calculated DFFs resulting from certain particle shapes for the cases of (a) aggregates of spherical grains with different fractal dimensions $D_{\mathrm{f}}$ and of (b) particles in the form of Gaussian random spheres (GRS, Muinonen et al. 1996) with different standard deviations $\sigma$ of the surface modulation. We may note here that GRS grain shapes are used in scattering models for large grains as well (e.g. Volten et al. 2001). However, this is an entirely different (ray optics) approach, where the size distribution is also reflected by the (often extreme) GRS shape. In contrast, in our small-particle-limit approach the GRS model describes only the grain shape.

The DFFs and illustrations of the particle morphologies are shown in Fig. 1. In both cases, the DFFs broaden strongly with variation of the respective parameter, i.e. when going from compact to fluffy (low-dimension) aggregates and from sphere-like to extremely structured GRS.

However, the details are different in the two series. While the "main peak" of the DFF shifts to higher $L$ with decreasing $D_{\mathrm{f}}$ in the case of the spherical grain aggregates, the opposite is the case for the GRS with increasing $\sigma$. For the extreme cases, namely the aggregate with $D_{\mathrm{f}}=1.2$ and the GRS with $\sigma=0.7$, these main peaks are located at $L=0.6$ and $L=0.05$, respectively. Consequently, we can expect the two types of DFFs to produce band profiles peaking on opposite sides of the sphere resonance position $(\operatorname{Re}(\varepsilon) \sim-2)$. Figure 2 demonstrates this for less extreme geometries, i.e. an aggregate with $D_{\mathrm{f}}=2.4$ and a GRS with $\sigma=0.3$. The short-wavelength peak of the aggregate particle spectrum $(\operatorname{Re}(\varepsilon) \sim-1.5)$ corresponds to $L \sim 0.4$, whereas the peak of the GRS spectrum $(\operatorname{Re}(\varepsilon) \sim-6)$ corresponds to $L \sim 0.15$. According to the DFF, it was expected to occur at $L \sim 0.1$. The difference probably stems from the influence of $\operatorname{Im}(\varepsilon)$, which increases towards longer wavelengths.

For the aggregates of spherical grains, there is actually another peak in the DFF, which becomes stronger and stronger with lowering $D_{\mathrm{f}}$, namely at $L=0$. This component of the DFF produces a long-wavelength shoulder in the band profile in addition to the short-wavelength peak (see Fig. 2). The $L=0$ component indicates two- or one-dimensional elongated, i.e. plate- or needle-like, structures. Agglomerates of spheres with point-like contacts are in principle not expected to produce such a component. Its presence is a consequence of the limited resolution of the discretization, which does not allow between point-like contacts and extended contact areas characteristic of e.g. coalesced particle aggregates to be distinguished (cf. Andersen et al. 2006). This is a direct effect of the method used to compute the DFFs (based on the discrete dipole approximation). However, coalescence of particles is often observed in real particulates, and its presence in the model distributions is not at all a shortcoming for their application, as we show.

In this paper, we make use of these DFFs by comparing simulated infrared extinction spectra for such synthetic particle shapes with the measured spectra of real particulates with known (different) shapes. We consider particulates composed of spinel, corundum, and forsterite (see next section), for which the wavelength-dependent optical constants $\varepsilon_{\mathrm{p}}(\lambda)$ are known from the literature. We use data by Fabian et al. (2001a) for synthetic stoichiometric spinel, Querry et al. (1985) for corundum, and Sogawa et al. (2006) for forsterite. For the last two, the cross sections obtained for the individual crystal axes are averaged. 

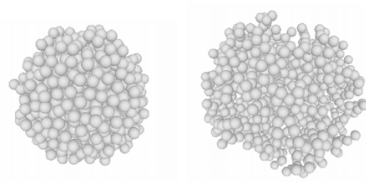

$D_{\mathrm{f}}=2.8$

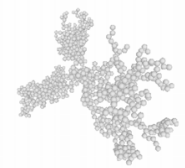

$D_{\mathrm{f}}=1.8$

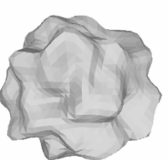

$D_{\mathrm{f}}=1.2$

$\sigma=0.1$

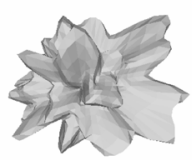

$\sigma=0.3$
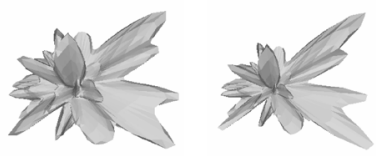

$\sigma=0.5$

$\sigma=0.7$
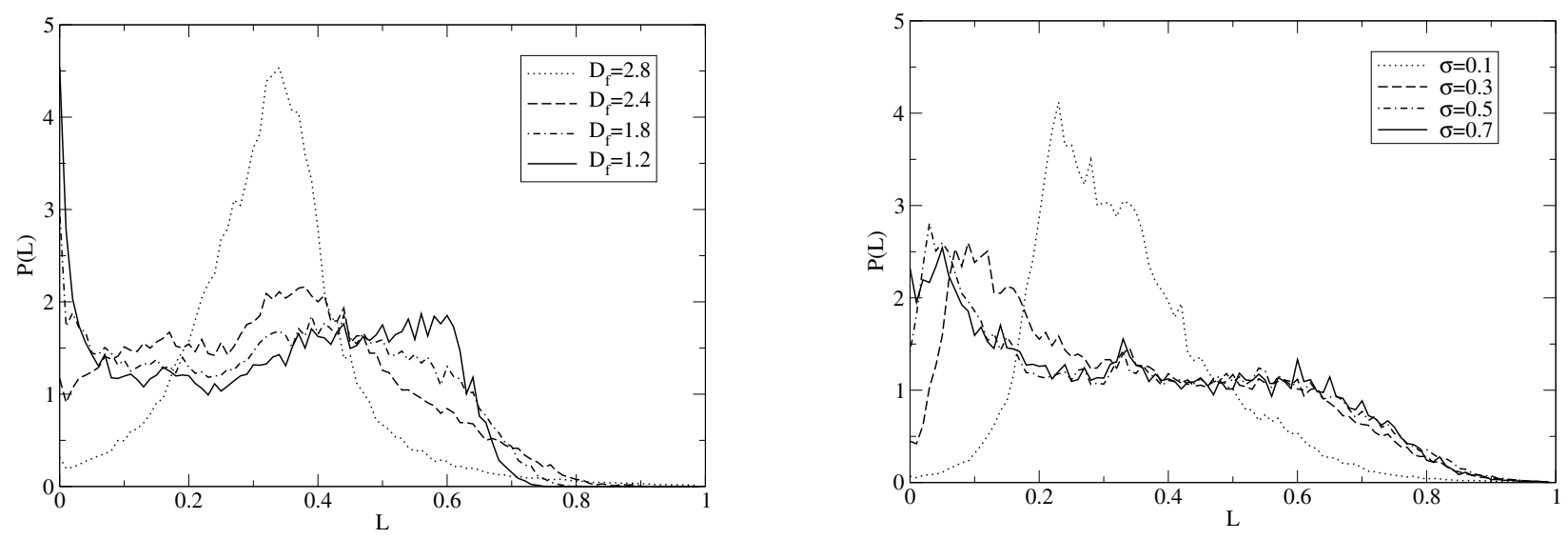

Fig. 1. Synthetic particle shapes and DFFs used in the modeling for aggregates of spherical grains with different fractal dimensions $D_{\mathrm{f}}(l e f t)$ and Gaussian Random Spheres with different standard deviations $\sigma$ (right).

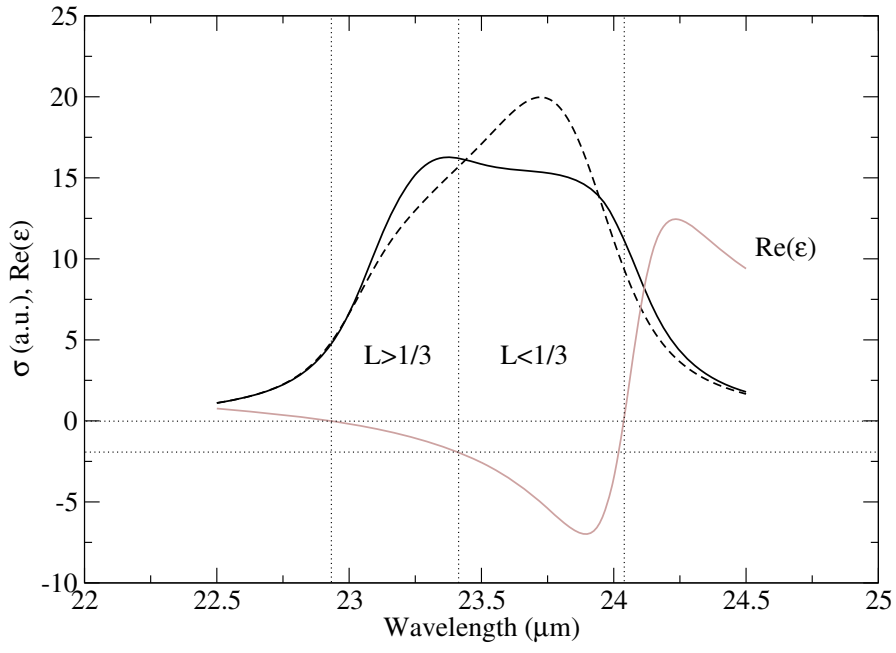

Fig. 2. Extinction spectra of fractal aggregates with $D_{\mathrm{f}}=2.4$ (solid black line) and Gaussian random spheres with $\sigma=0.3$ (dashed line) calculated for a Lorentzian oscillator type dielectric function. $\operatorname{Re}(\varepsilon)$ is also shown (gray line). The vertical lines indicate the wavelength range where $\operatorname{Re}(\varepsilon)=1-1 / L$ can be fulfilled and the position of the sphere resonance $(\operatorname{Re}(\varepsilon)=-2)$.

For the environment, the dielectric constant $\varepsilon_{\mathrm{e}}$ is unity in the simulation of aerosol spectra, 2.31 in cases when the particles are embedded into $\mathrm{KBr}$ matrices, and 3.03 for the case of embedding into a CsI matrix.

\section{Experimental spectra}

The experimental setup for the measurement of infrared extinction spectra of dust particles dispersed in air (aerosol measurements) was described by Tamanai et al. (2006a,b, 2009a,b). For comparison with the simulations based on the DFF model, we have selected two samples of each of the three minerals spinel $\left(\mathrm{MgAl}_{2} \mathrm{O}_{4}\right)$, corundum $\left(\mathrm{Al}_{2} \mathrm{O}_{3}\right)$, and forsterite $\left(\mathrm{Mg}_{2} \mathrm{SiO}_{4}\right)$, from these papers. The sample properties are listed in Table 1 .

The two samples of each material have significantly different grain shapes. In general, one of the particulates can be characterized by a roundish shape or at least by having round edges, which is often the case for particles that have been condensed from the gas or liquid phase in a relatively fast process, whereas the other particulate is characterized by an irregular grain shape with sharp edges, which is typical of particulates produced by crushing larger pieces of material, but may also result from slow crystal growth. The spectra of the two classes of particulates differ significantly, as discussed by Tamanai et al. (2006b, 2009b). Correspondence with astronomically observed infrared dust emission bands has been found to be better in some cases for the roundish shapes, especially for the oxides in AGB star outflows (Tamanai et al. 2009b; see also Posch et al. 1999; and Fabian et al. 2001a), but in other cases better for the irregular shapes, especially for forsterite (Tamanai et al. 2006b; see also Molster et al. 2002; Fabian et al. 2001b). Particle shapes and spectra are shown in Fig. 3.

\section{Results}

\subsection{Simulated spectra vs. measured aerosol spectra}

Figure 4 shows the comparison of the simulated spectra for spinel, corundum, and forsterite particles with the measured spectra. The left column compares the spectra calculated for the aggregates of spheres to the measured spectra of the roundish particulates. The simulated band profiles consist in most cases of peaks at short wavelengths and shoulders at longer wavelengths (compare Fig. 2). The latter strengthen with lowering fractal dimension of the aggregate, as do the $L \sim 0$ components in the DFFs. The "primary" peak at shorter wavelengths, which 
Table 1. Properties of the samples.

\begin{tabular}{ccccccc}
\hline \hline Material & Chemical formula & Product info & Processing & Grain size & Grain shape & Reference \\
\hline Spinel & $\mathrm{MgAl}_{2} \mathrm{O}_{4}$ & Aldrich & & $<0.1 \mu \mathrm{m}$ & round & T09b, CSp2 \\
& & Alfa Aesar & Sedimentation & $<1 \mu \mathrm{m}$ & irregular & T09b, CSP1 \\
Corundum & $\mathrm{Al}_{2} \mathrm{O}_{3}$ & Alfa Aesar & & $<0.3 \mu \mathrm{m}$ & ellipsoidal & T09b, CAC1 \\
& & GC Jena & Sedimentation & $<2 \mu \mathrm{m}$ & irregular & T09b, CAC2 \\
\multirow{2}{*}{ Forsterite } & $\mathrm{Mg}_{2} \mathrm{SiO}_{4}$ & Marusu & & $<0.3 \mu \mathrm{m}$ & ellipsoidal & T06b, CF2 \\
& & Alfa Aesar & Sedimentation & $<1 \mu \mathrm{m}$ & irregular & T06b, CF1 \\
\hline
\end{tabular}
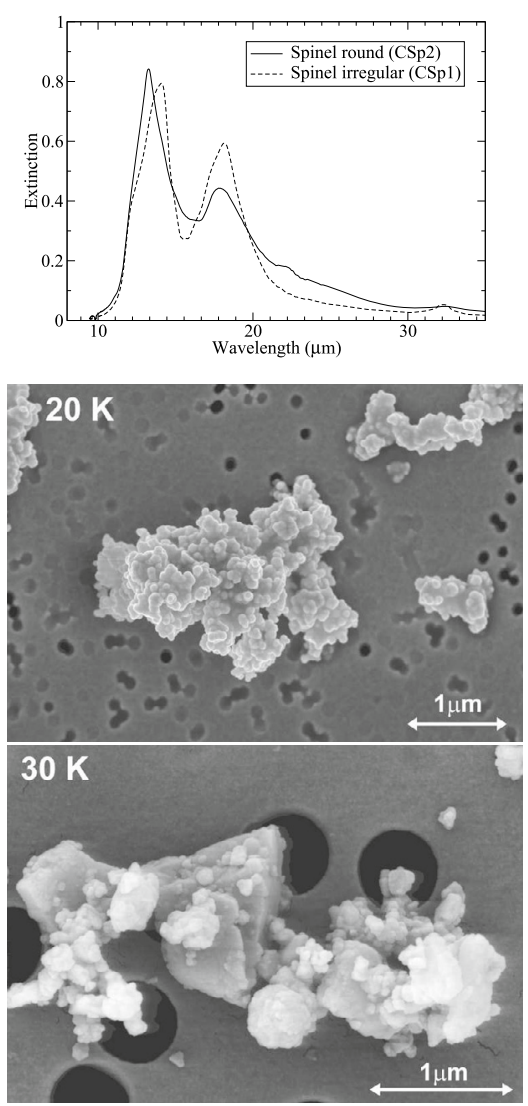
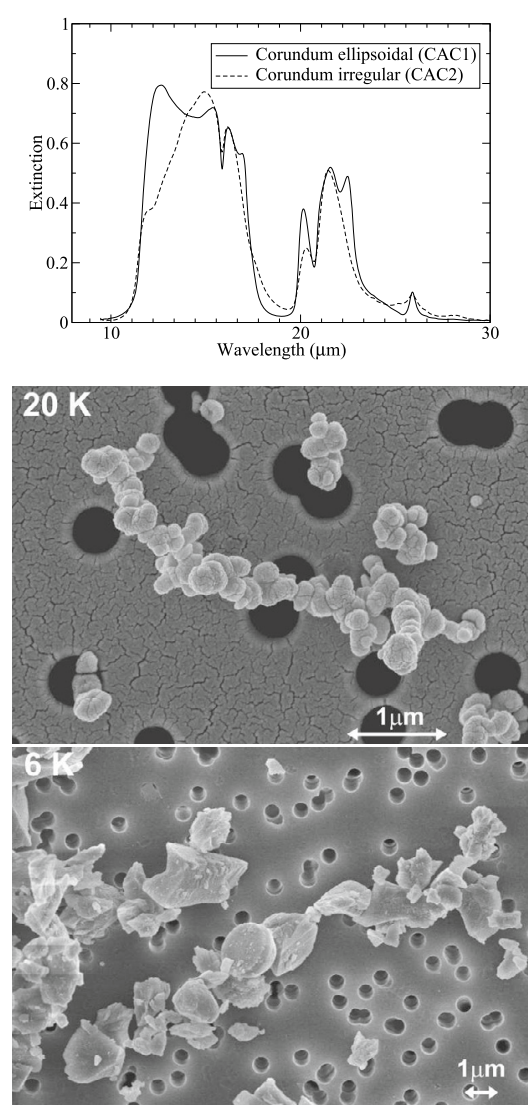
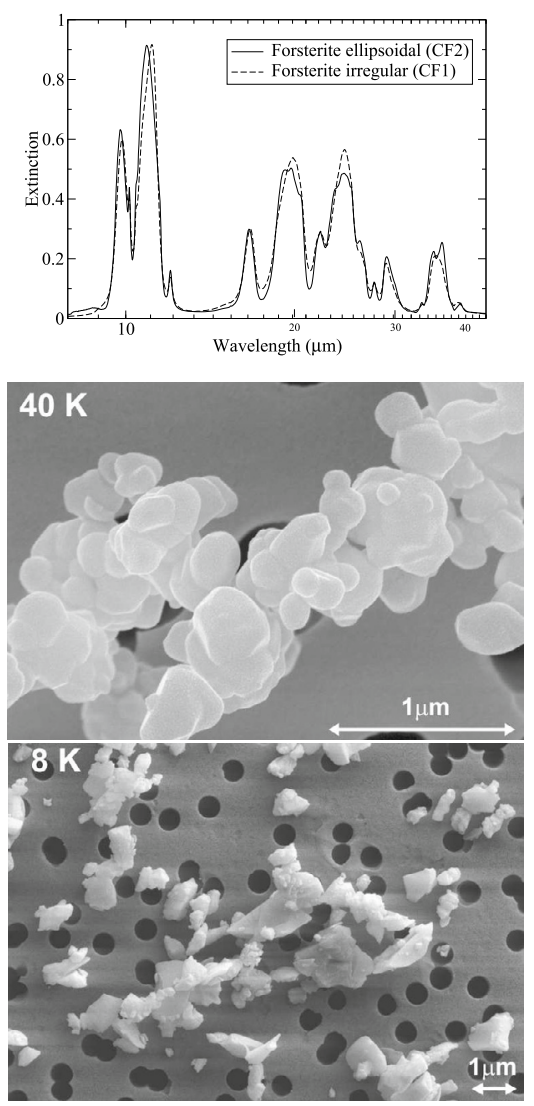

Fig. 3. Aerosol infrared extinction spectra and SEM micrographs of the spinel (left), corundum (middle), and forsterite (right column) particulates. The solid lines are the spectra of the particulates with roundish shapes, which are shown in the images in the second row. The dashed lines correspond to the spectra of the sharp-edged grains (images in bottom row). The dark round filter holes in the image background are about $0.5 \mu \mathrm{m}$ in diameter except for the image of the roundish spinel sample (upper left micrograph), where they are $0.1 \mu \mathrm{m}$ in diameter. The numbers indicate SEM magnification.

weakens only between $D_{\mathrm{f}}=2.8$ and 2.4 , remains present while shifting to shorter wavelengths. This often leads to a "rectangular" band profile for low fractal dimensions, such as at $D_{\mathrm{f}} \leq 1.8$, which actually reproduces the measured spectrum of the corundum sample well. The comparison with the other simulated spectra demonstrates clearly that in the case of these particles a strong long-wavelength band component is definitely required to fit the measured profiles at about $17 \mu \mathrm{m}$ and $22.5 \mu \mathrm{m}$. The finding that a fractal dimension of $D_{\mathrm{f}}=1.8$ provides the best match corresponds nicely to the observed agglomeration state of the corundum powder, which is mainly chain-like (see Fig. 3). For the other samples of roundish grain shapes, the long-wavelength component is weaker but also present. For both the spinel and the forsterite samples, the $D_{\mathrm{f}}=2.4$ spectrum fits quite well to the peaks in the measured spectra.
In the case of spinel, the region in between the two bands and longward of $20 \mu \mathrm{m}$ shows an enhanced extinction that is not reproduced by any of the calculations. We interpret this feature as being caused by extinction from clumps of particles. Apparently, due to the small size of the individual grains, the clumps appear compact enough to act partially similar to large grains. According to Mie calculations, grains of about $5 \mu \mathrm{m}$ size already provide sufficient extinction (absorption and scattering) at these wavelengths.

Moreover, we note that a few bands, such as the forsterite $10 \mu \mathrm{m}$ and $27.5 \mu \mathrm{m}$ and the corundum $20 \mu \mathrm{m}$ bands, are not satisfactorily reproduced by the same models as the majority of the bands. This is discussed in Sect. 4.2.

The right column compares the spectra of the irregular particulates with the simulations for the GRS of different surface 

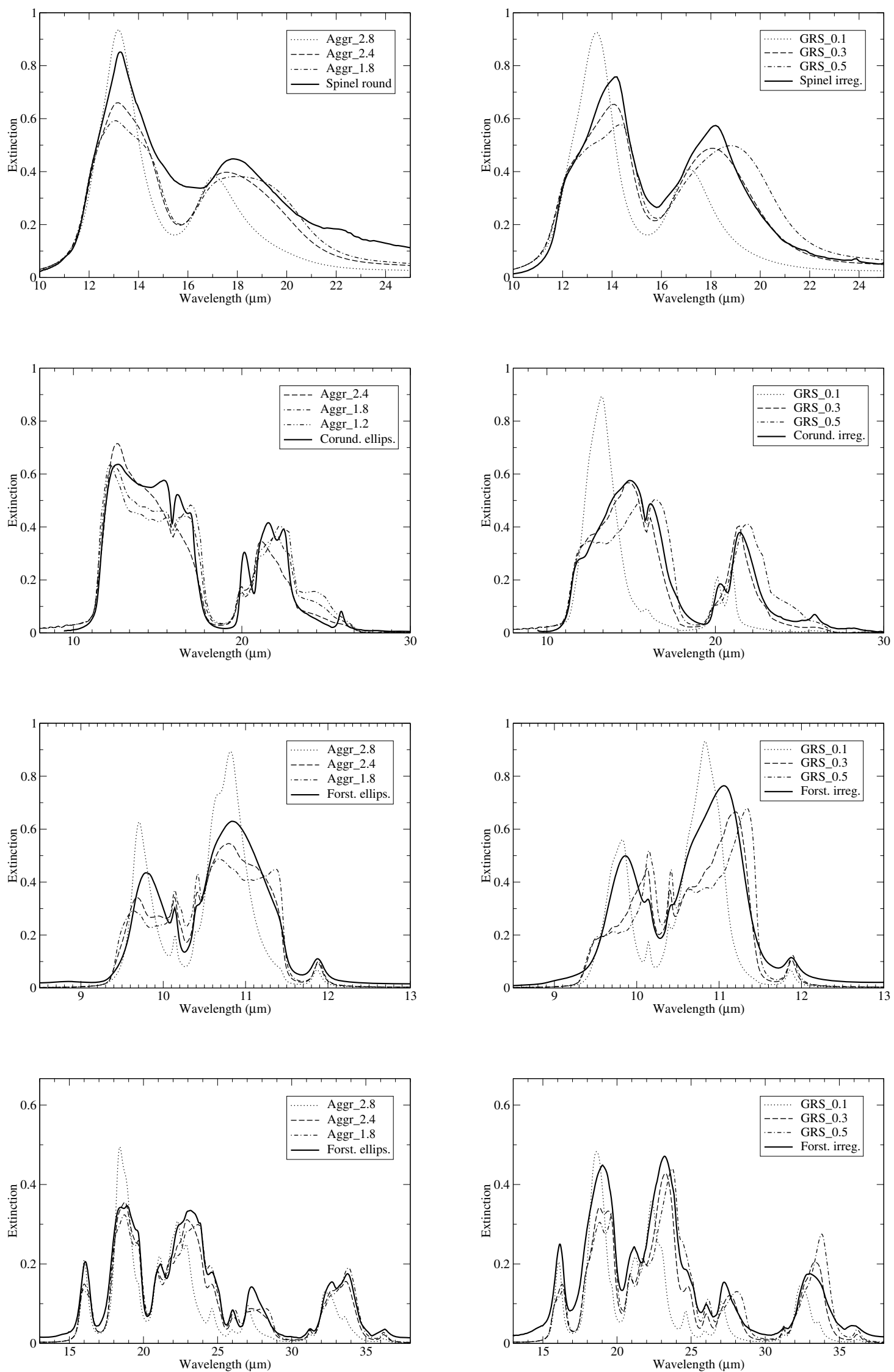

Fig. 4. Comparison of measured (solid lines) and simulated extinction spectra for the roundish (left column) and irregular (right) particle shapes. DFF models used in the simulations are given in the legends. The forsterite spectra have been divided into two wavelength ranges. 


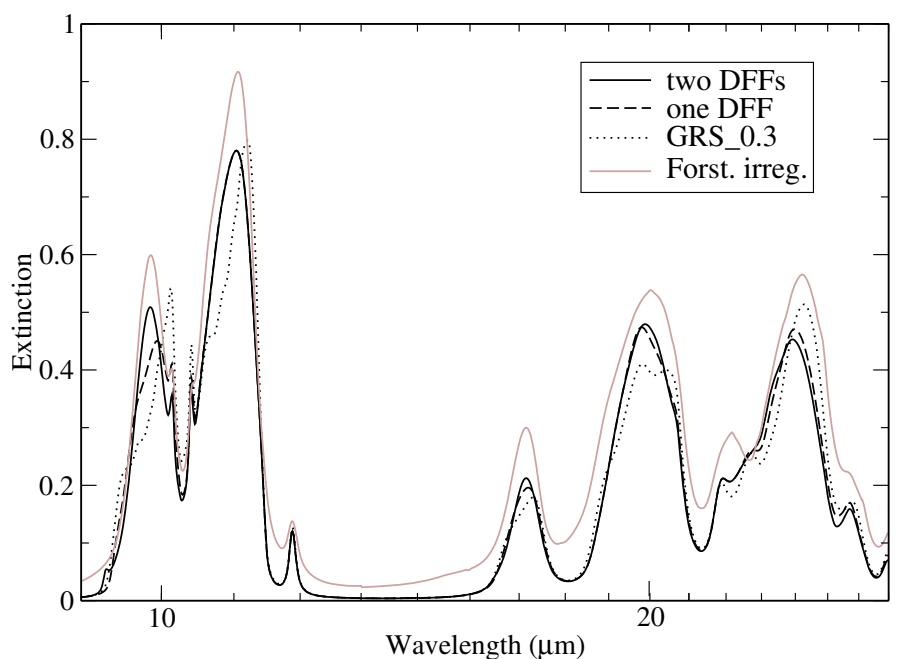

Fig. 5. Comparison of fits to the measured spectrum (gray line) of irregular forsterite particles using a single DFF for the contributions of all crystal axes (dashed line) and allowing two DFFs, i.e. a separate DFF for the contribution of the vibrational modes along the crystallographic $x$-axis (solid line). The DFFs obtained by the two-DFF fitting are plotted in Fig. 6. For comparison, the simulation using the GRS model with $\sigma=0.3$ is also shown (dotted line).

modulation. As expected, the band profiles in these simulated spectra are dominated by peaks that shift continuously to larger wavelengths with an increasing standard deviation parameter. A shoulder remains at the short-wavelength edge of the profiles. The comparison with the measured spectra reveals excellent agreement for the $\sigma=0.3$ simulation in the cases of spinel and corundum (again apart from the $\lambda=20 \mu \mathrm{m}$ band). For the forsterite, $\sigma=0.3$ is still the best-fitting calculated spectrum, even if it seems that a little lower $\sigma$ may have given a better match. This is especially true for the $10 \mu \mathrm{m}$ band, which we discuss separately in Sect. 4.2.

\subsection{Fitting of DFFs and the problem of anisotropy}

An alternative way to compare theory and measurement is to fit DFFs to the measured spectra, hoping for a better reproduction of the measurements and aiming at the comparison of the obtained DFFs. We have developed an automatic fitting procedure that is able to handle anisotropic materials, i.e. contributions of up to three dielectric functions (in the case of forsterite, two for corundum) and even with the option of using different DFFs for each of these. In Sect. 4.3, we show the result of this fitting for the irregular spinel. The fitting worked without problems for this isotropic material.

In the previous section, we noted that a few bands in the spectra of the anisotropic materials show poor reproduction by the models, such as the $10 \mu \mathrm{m}$ band of forsterite and the $20 \mu \mathrm{m}$ band of corundum. For reproducing them, a DFF peaking strongly between $L=0.2$ and 0.25 (close to that of GRS 0.1 ) would work best, but it would not reproduce the other bands. These bands belong to vibrational modes along certain crystal axes, so introducing different DFFs for different crystal axes could solve these problems.

In general, deviations in the DFFs along different crystal axes are not an unlikely option, because the grains could be primarily elongated along certain crystal axes. Such grain shapes can be produced both by growth and shattering processes. Allowing for

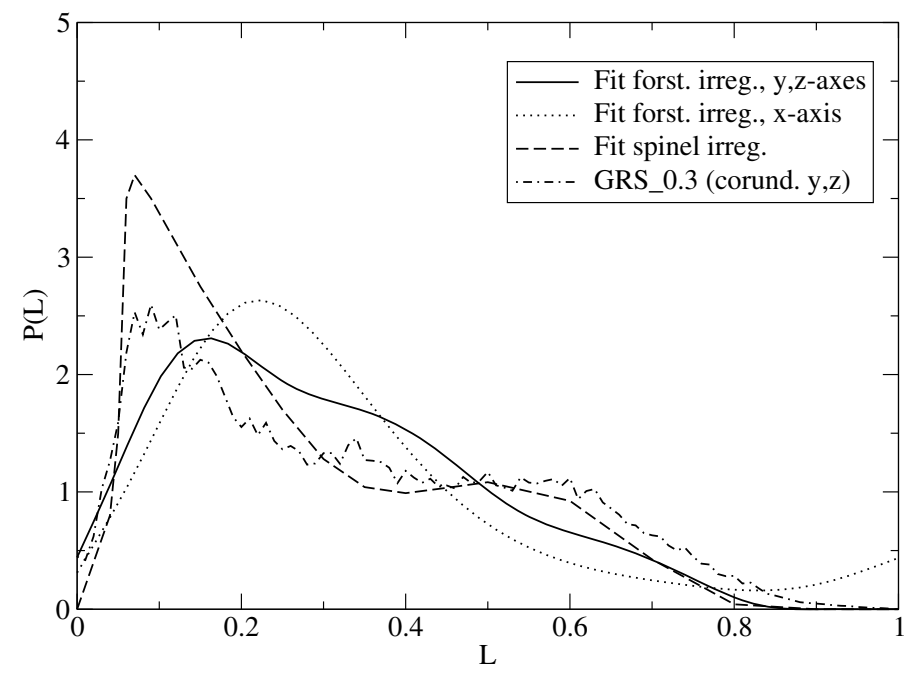

Fig. 6. Comparison of the fitted DFFs for irregular forsterite particles and irregular spinel particles (dashed line) with the GRS model with $\sigma=0.3$ (dash-dotted), which provides an excellent fit for the spectrum of the irregular corundum particles.

different DFFs for the different crystal axes lead to a satisfactory reproduction of the problematic bands both for forsterite and for corundum. Figure 5 shows as an example the fits for the irregularly shaped forsterite with (a) only one DFF for all axes and (b) a separate DFF for the contributions of the vibrations along the crystallographic $x$-axis. The better match in the $10 \mu \mathrm{m}$ band is clearly visible.

However, the DFFs for the different axes needed to be very different. The DFF required by the problematic bands were always similar, with a narrow peak in the $L=0.2-0.3$ range. This changed only slightly for roundish grains, even if the main DFF peaked then on much higher $L$ values. In Fig. 6, the two fitted DFFs are shown for the irregular forsterite particles (see Fig. 5), where the difference is still quite moderate. The GRS model with $\sigma=0.3$, however, represents the case well for the $y$ and $z$-axis DFF for the irregular corundum particles. Here, the difference from the $x$-axis DFF is very strong. Especially for the roundish grains, such extreme differences between the crystal axes and the nondependence on the grain-shape seemed unlikely. In some bands, we noted that the poor fit introduced by the additional DFF had to be compensated for by the other components.

This pointed to a different reason for the mismatch in some bands, which turned out to be an incorrectness in the model, namely in the treatment of the anisotropy of the materials. The simple averaging of the absorption cross sections calculated for the crystallographic axes is correct for spheres that are small compared to the wavelengths and for ellipsoids, if the ellipsoid principal axes are associated with the crystal axes. When assuming complicated shapes, such as the GRS or aggregates, however, it is clear that this condition is not maintained. Here, the influence of the polarization along one axis on the excitation along other axes has to be considered.

This can be demonstrated by means of the discrete dipole approximation (DDA) model. The DDA allows one to take the full tensor nature of the refractive index into account. We will demonstrate the effects of this in a separate paper (Min et al., in prep). 


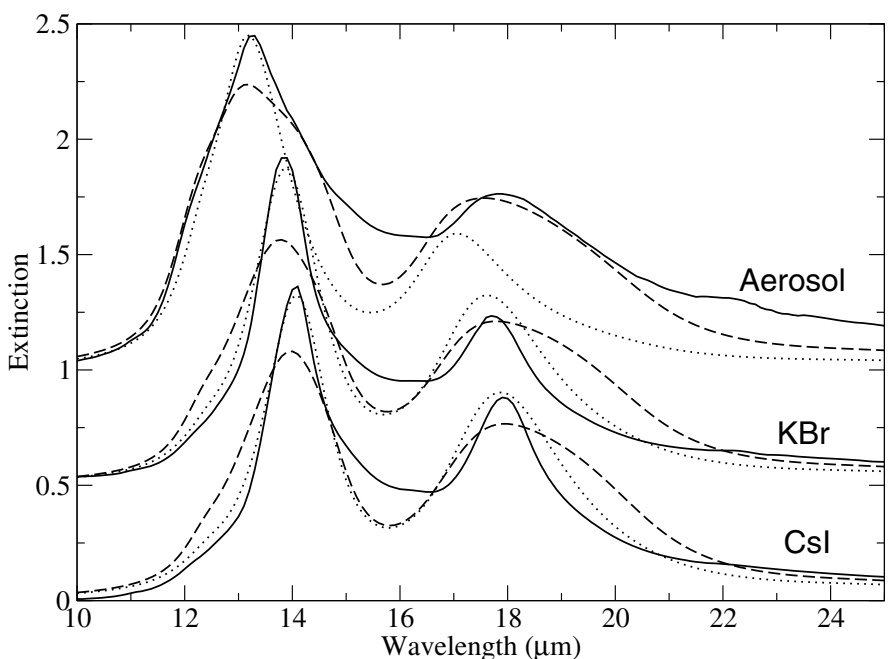

Fig. 7. Comparison of measured (solid lines) and simulated extinction spectra for roundish spinel particles embedded in different media. The DFF models used are the aggregates of spherical grains with $D_{\mathrm{f}}=2.8$ (dotted lines) and $D_{\mathrm{f}}=2.4$ (dashed lines).

\subsection{Reproduction of $\mathrm{KBr}$ and $\mathrm{Cs}$ spectra}

An interesting question is whether the DFF models can predict the influence of embedding media on the spectra of particulates. Tamanai et al. (2006b, 2009b) demonstrated the strong influence of potassium bromide and cesium iodide matrices used in the conventional pellet technique onto IR band profiles of embedded particulates. Within the DFF model, the embedding medium is taken into account simply via the relative dielectric constant $\varepsilon=$ $\varepsilon_{\mathrm{p}} / \varepsilon_{\mathrm{e}}$.

Figures 7 and 8 show simulated spectra for spinel using identical DFFs and different dielectric constants of the embedding medium $\left(\varepsilon_{\mathrm{e}}=1\right.$ for aerosol, 2.31 for $\mathrm{KBr}$, and 3.03 for CsI) compared to corresponding measured spectra. For the irregularly shaped particles (Fig. 7), the DFF for GRS with $\sigma=0.3$ and a fitted DFF were used. The comparison shows that the former, which reproduces the aerosol measurements satisfactorily, gives a still quite imprecise representation of the measured spectra for embedded particles. However, this does not mean that the model fails to treat the influence of the embedding, because some adjustment of the DFF (see Fig. 6) leads to a nearly perfect fit of all the spectra measured in different matrices.

Unfortunately, this is not the case for the roundish particles (Fig. 7, see especially the $18 \mu \mathrm{m}$ band). Here, the embedded particles are best matched by a DFF strongly dominated by the spherical character of the single grains or characterized by very compact aggregation, whereas for the aerosol spectra we had to use a DFF calculated for a lower fractal dimension of the aggregates. If this indeed reflects different morphologies of the spinel particles in aerosol and in embedded states, it could possibly indicate a compaction of the aggregates by the pellet pressing, or, since we have already noted that these spectra are also influenced by size effects, an aggregate size reduction by the thorough mixing with the matrix powder. The size of the aggregates of course could always be influenced by the preparation technique. Unfortunately, an inspection of the morphology of the embedded aggregates is not possible and a treatment of size effects is beyond the capabilities of this theory. For the other samples, which have larger grains and therefore show less clumping, we have not observed similar effects.

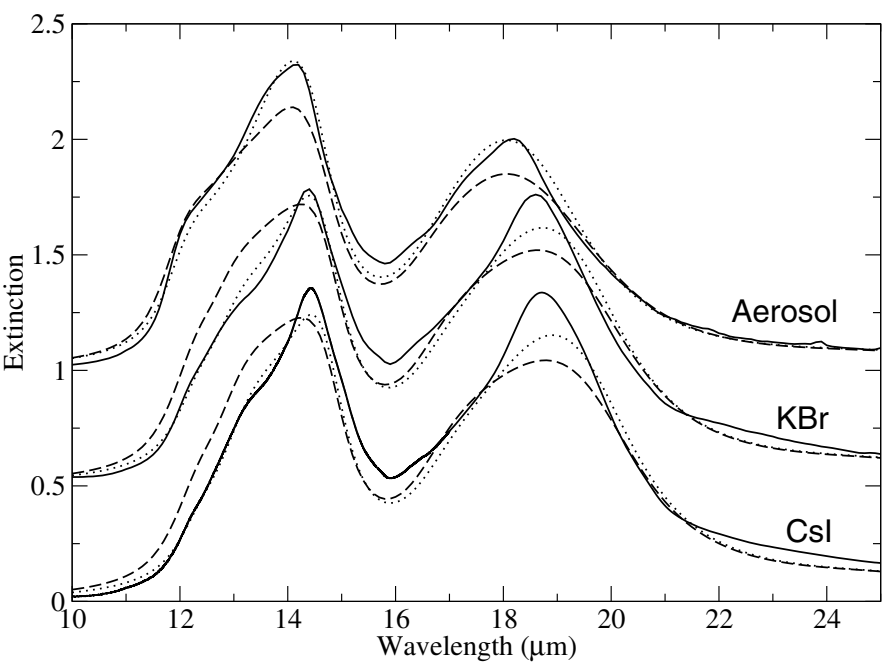

Fig. 8. Same as Fig. 7 for irregular spinel particles. The DFF models used here are the Gaussian random spheres with $\sigma=0.3$ (dashed lines) and a fitted DFF (see Fig. 6).

\section{Conclusions}

By comparison with experimental spectra, we have demonstrated the usefulness of the DFF approach for simulating the infrared extinction spectra of submicron-sized particulates composed of oxide and silicate minerals. Predefined DFFs with certain characteristics can be used to achieve a satisfactory prediction for the shape dependence of the measured infrared band profiles. Good agreement between experimental and calculated band profiles was achieved by using

1. DFFs calculated for Gaussian random spheres with a surface modulation of $\sigma \sim 0.3$ in the case of particulates with irregular grain shapes. The main characteristics of these DFFs is a strong and relatively sharp peak at $L \sim 0.1$.

2. DFFs calculated for aggregates of spheres with a fractal dimension $D_{\mathrm{f}}=2.4$ or $D_{\mathrm{f}}=1.8$ in the case of particulates with roundish grain shapes. These DFFs peak at $L=0.33-0.45$ and also have a component at very low $L$ values, which is required to fit the experimental band profiles and seems to be correlated with the agglomeration state in the particulate.

Our results suggest that DFF models, such as the ones for GRS with $\sigma \sim 0.3$ and fractal aggregates with $D_{\mathrm{f}}=2.4$ or $D_{\mathrm{f}}=1.8$, would reflect morphological properties of real particles significantly better than CDE or DHS models, so would be useful in the simulation of cosmic-dust spectra. This could lead to better predictions of infrared band profiles or at least to a valid estimate of possible band variations. A necessary condition is that grain sizes that are small compared to the wavelength can be assumed to dominate the spectrum.

We recognized that the averaging of spectra obtained by the DFF model for the individual crystallographic orientations of an anisotropic material is incorrect and leads in some cases to significantly wrong band profiles. This is a problem of all comparable theories and can only be overcome by exact simulations, such as a full DDA treatment, which however lacks the statistical approach and is difficult to implement in comprehensive astronomical spectroscopic simulations. Therefore, currently a safer way may be to rely on aerosol-measured spectra.

Acknowledgements. This work was supported by Deutsche Forschungsgemeinschaft under grant Mu 1164/6-1. Special thanks go to W. Teuschel 
and G. Born for their help with the experiments. We are grateful to Prof. C. Koike for providing the Marusu forsterite sample. Furthermore, we thank the Elektronenmikroskopisches Zentrum of the medical faculty of the Friedrich Schiller University Jena for help with the SEM imaging. H.M. acknowledges support by the International Space Science Institute (ISSI) in Bern, Switzerland ("Exozodiacal Dust Disks and Darwin" working group, http: //www. issibern.ch/teams/exodust/)"

\section{References}

Andersen, A. C., Mutschke, H., Posch, Th., Min, M., \& Tamanai, A. 2006, J. Quant. Spectr. Rad. Transfer, 100, 4

Bohren, C. F., \& Huffman, D. R. 1983, Absorption and Scattering of Light by Small Particles (New-York: J.Wiley \& Sons)

Fabian, D., Posch, Th., Mutschke, H., Kerschbaum, F., \& Dorschner, J. 2001a, A\&A, 373, 1125

Fabian, D., Henning, T., Jäger, C., et al. 2001b, A\&A, 378, 228

Lisse, C. M., VanCleve, J., Adams, A. C., et al. 2006, Science, 313, 635

Lisse, C. M., Beichman, C. A., Bryden, G., \& Wyatt, M. C. 2007, ApJ, 658, 584
Min, M., Hovenier, J. W., \& de Koter, A. 2005, A\&A, 432, 909

Min, M., Hovenier, J. W., Dominik, C., de Koter, A., \& Yurkin, M. A. 2006, J. Quant. Spectr. Rad. Transfer, 97, 161

Molster, F. J., Waters, L. B. F. M., Tielens, A. G. G. M., Koike, C., \& Chihara, H. 2002, A\&A, 382, 241

Muinonen, K., Nousiainen, T., Fast, P., Lumme, K., \& Peltoniemi, J. I. 1996, J. Quant. Spectr. Rad. Transfer, 55, 577

Posch, T., Kerschbaum, F., Mutschke, H., et al. 1999, A\&A, 352, 609

Querry, M. R. 1985, Technical Library CRDC-CR-85034, Chem. Research and Development Center, US Army AMC Command

Sogawa, H., Koike, C., Chihara, H., et al. 2006, A\&A, 451, 357

Tamanai, A., Mutschke, H., Blum, \& J., Neuhäuser, R. 2006a, J. Quant. Spectr. Rad. Transfer, 100, 373

Tamanai, A., Mutschke, H., Blum, J., \& Meeus, G. 2006b, ApJ, 648, L147

Tamanai, A., Mutschke, H., Blum, J. 2009a, in Cosmic Dust - Near and Far, ed. Th. Henning, E. Grün, \& J. Steinacker, ASP Conf. Ser., accepted

Tamanai, A., Mutschke, H., Blum, J., et al. 2009b, A\&A, 501, 251

van Boekel, R., Min, M., Leinert, Ch., et al. 2004, Nature, 432, 479

Volten, H., Muñoz, O., Rol, E., et al. 2001, J. Geophys. Res., 106(D15), 17, 375 